\title{
THE RELIABILITY AND ACCURACY OF RADIOGRAPHS IN THE ASSESSMENT OF PEDICLE SCREW PLACEMENT; A COMPARISON WITH COMPUTERIZED TOMOGRAPHY
}

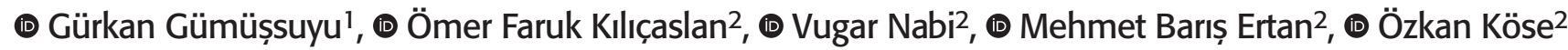 \\ ${ }^{1}$ Istinye University Faculty of Medicine, Department of Orthopaedics and Traumatology, İstanbul, Turkey \\ ${ }^{2}$ Antalya Training and Research Hospital, Clinic of Orthopaedics and Traumatology, Antalya, Turkey
}

\begin{abstract}
Objective: The purpose of this study was to evaluate the reliability and accuracy of radiographic assessment of pedicle screw placement, using computed tomography (CT) as the reference standard.

Materials and Methods: Fifteen patients who underwent posterior spinal fusion were retrospectively reviewed. Pedicle screw position was rated using postoperative anteroposterior and lateral radiographs by two independent spinal surgeons twice at least four weeks apart. CT was rated by two other spinal surgeons collectively and reached a consensus decision. Kappa analysis was used to measure the relative agreement between radiographic ratings and $\mathrm{CT}$ evaluation.

Results: A total of 367 screws were evaluated. According to CT evaluation, 69 (18.8\%) pedicle screws were determined as malpositioned. However, in plain radiographs, $53(14.4 \%)$ pedicle screws were rated as malpositioned. The intraobserver reliability of radiographic ratings was almost perfect for both observers at both times (ICC $=0.833$ and 0.817 ). On the other hand, the interobserver reliability was substantial at both occasions $($ ICC $=0.722$ and 0.808$)$. The agreement between radiographic ratings and CT evaluation (reference standard) was substantial (kappa=0.686, $95 \%$ confidence interval $=0.586-0.785$ ). The sensitivity and specificity of radiographs to detect a malpositioned screw were $65.2 \%$ and $97.3 \%$, respectively.

Conclusion: Radiographic ratings showed substantial and almost perfect agreement among observers. However, the accuracy of radiography was low compared to CT evaluation. In case of suspicion, CT should be the choice of the imaging modality to decide on a malpositioned screw. Keywords: Screw malposition, plain radiographs, CT reconstruction, accuracy
\end{abstract}

\section{INTRODUCTION}

Pedicle screws have become the gold standard fixation method in spinal surgery due to their various advantages, such as greater rotational stability, the possibility of instrumentation in the absence of posterior elements, diminished number of levels required for stability, avoidance of neural dissection, and shorter operation time ${ }^{(1)}$. However, these screws are not entirely secure and might result in severe neurovascular and visceral injuries in case of extra-pedicular placement ${ }^{(2,3)}$. Although various assistive methods are used to increase the accuracy of screw position, the pedicle screw malposition still remains a significant problem. In a recent metanalysis that examined 51,161 pedicle screws, the accuracy of pedicle screws ranged between $61.3 \%$ to $100 \%$ using different guided techniques ${ }^{(4)}$. In general, five different incorrect screw placements can occur. The first one is the perforation of the anterior cortex. In this malposition, mortal injuries may occur because the tip of the screw might damage the vascular and visceral organs lying anterior to the vertebral corpus ${ }^{(5-7)}$. The second is the perforation of the medial wall of the pedicle. In this case, the neural structures are under the risk of injury. Another malposition is perforation of the lateral pedicular wall. Lateral perforations may result in root injuries or visceral injuries such as pneumothorax in the thoracal spine ${ }^{(8)}$. In addition, screws can penetrate the superior or inferior disc space, which may cause chronic pain. All these incorrect screw placements not only result in significant injuries but also cause decreased fixation strength, insufficient deformity correction and screw pull-out, as the stability is also impaired ${ }^{(9,10)}$.

Computerized tomography (CT) is currently accepted as the goldstandard method to evaluate the position of the pedicle screws in post operative imaging. However, post operative radiographs are the first-line imaging modality in evaluating pedicle screw placement which is both practical, inexpensive, and safe. Due to the complexity of the spine anatomy, it is not always easy 
to decide on the placement of a pedicle screw on radiographs. Few studies in the literature examine the reliability and accuracy of radiological evaluation of pedicle screw placement. We hypothesized that radiographic evaluation underestimates the pedicle screws' malposition and cannot precisely diagnose screw malposition. This study aimed to test the reliability of the radiological criteria used in the assessment of the pedicle screw placement and compare it with the gold standard CT examination.

\section{MATERIALS AND METHODS}

\section{Study Population}

This study was performed on patients who underwent posterior spinal fusion using pedicle screw instrumentation between 2018 and 2020 in authors' institutions. Patients who had both postoperative anteroposterior (AP) and lateral spinal radiographs (scoliosis view) and $\mathrm{CT}$ were identified and included in the study. $\mathrm{CT}$ is not a routine imaging method used for postoperative radiological evaluation in our clinic. CT is ordered only in suspicious cases, such as the development of a postoperative neurological deficit or suspicious direct radiographic findings compatible with screw malposition. Clinical and demographic data were obtained from institutional medical records, and imaging studies were obtained from the picture archiving and communication system (PACS). This study was carried out in accordance with the ethical standards laid down in the 1964 Declaration of Helsinki and its later amendments, and the Institutional Review Board approved the study protocol (approval date/issue: 04.03.2021/1-45).

\section{Sample Size Calculation}

The sample size was calculated to detect a difference in the Cohen's kappa statistics between minimum acceptable kappa

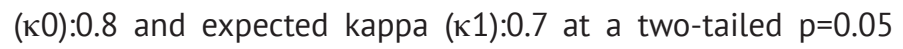
significance level ( $\alpha$ error) and with $80 \%$ power (1- $\beta$ error), assuming that two observers $(\kappa=2)$ rated each pedicle screw. A minimum number of 283 ratings (pedicle screws) were required $^{(11)}$. Among eligible patients, 15 patients with 367 pedicle screws were randomly selected and included in the study to obtain sufficient statistical power.

\section{Plain Radiographic Assessments}

Two independent spinal surgeons (Observer A \& Observer B) with at least five years of experience in spinal surgery took part in the study. Each observer independently rated radiographs on two separate occasions ( $\mathrm{t} 1$ and $\mathrm{t} 2$ ), at least four weeks apart. Observers were blinded to their previous readings. The order of the X-rays was randomized using a sequential, random number generator to prevent possible recall. All assessments were performed on digital radiographs that were stored in PACS using the software program Sectra IDS7 (Ver. 18.2., Sectra $A B$, Sweden) on the digital workstation. Five previously described criteria were used to evaluate the malposition of the pedicle screws (Figure 1$)^{(12,13)}$. Pedicle screws that met

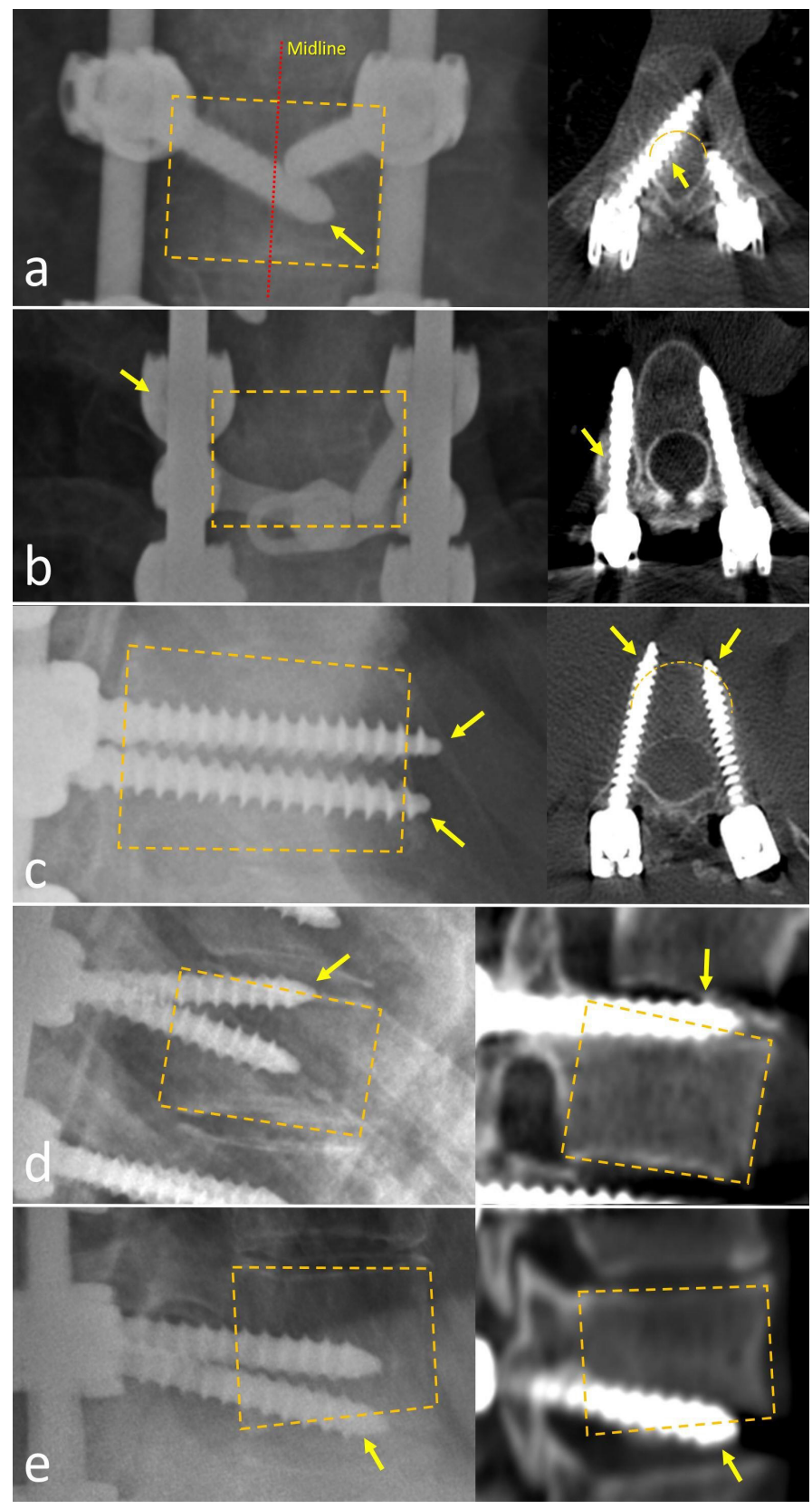

Figure 1. Five criteria used to assess the pedicle screw malposition on AP and lateral radiographs and representative CT images.

a) Medial perforation. The pedicle screw should be within the elliptic pedicle image and the tip of the screw should not pass beyond the midline. b) Lateral perforation. The tip of the pedicle screws should stay within the rectangle forming vertebral body and shouldn't deviate abnormally in the lateral direction (Yellow rectangle shows the vertebral corpus). c) Anterior cortical penetration. In the lateral radiography screws should stay within the borders of rectangle forming vertebral body and tip of the screws shouldn't exceed anterior cortex of vertebral corpus. d) Superior penetration. In the lateral radiography screws should stay within the borders of rectangle forming vertebral body and tip of the screws shouldn't exceed superior cortex of vertebral corpus. e) Inferior penetration. In the lateral radiography screws should stay within the borders of rectangle forming vertebral body and tip of the screws shouldn't exceed inferior cortex of vertebral corpus. 
turkishspine

any of these criteria were considered incorrectly placed. After the completion of ratings, the observers reached a consensus through discussion on their disagreements. The joint decision on radiographic ratings was used for comparison to $\mathrm{CT}$ ratings.

\section{CT Assessments}

Since $C T$ is the best available imaging technique for assessing the pedicle screw placement, the CT was used as a reference standard. CT evaluation was performed by two different observers (Observer C and observer D), except for those who evaluated direct radiography. These observers were also spinal surgeons. Both observers evaluated the CT collectively and reached a consensus decision. During the $\mathrm{CT}$ evaluation, they used the axial sections and the coronal and the sagittal views to improve accuracy. Besides, they performed multiplanar reconstruction (MPR) in suspicious screws. Similar criteria were used for $\mathrm{CT}$ ratings. Observers determined whether the screw was correctly positioned and if the screw was malpositioned, they also decided on the direction of the breach. The study design is illustrated in Figure 2.

\section{Statistical Analysis}

Statistical analysis was performed using SPSS Statistics Base v.23 for Windows. Descriptive statistics of the continuous and categorical data were presented as mean \pm standard deviation, range, and frequency distribution. Kappa statistics were used to establish a relative level of agreement on the categorical variables. Interpretation of the data was performed according to Landis and $\mathrm{Koch}^{(14)}$. Agreement was graded as slight $(\kappa=0$ $0.2)$, fair $(\kappa=0.21-0.40)$, moderate $(\kappa=0.41-0.60)$, substantial $(\kappa=0.61-0.80)$, and almost perfect $(\kappa=0.81-1)$.

\section{RESULTS}

There were 15 patients ( 3 male, 12 female) with a mean age of $29.2 \pm 19.9$ years (range: $13-73$ ). The etiologies were adolescent idiopathic scoliosis $(n=4)$, adult scoliosis $(n=5)$, neuromuscular scoliosis $(n=2)$, Scheuermann kyphosis $(n=2)$, and adult degenerative scoliosis $(n=2)$. An average of 15 segments (range: 10-17) was instrumented, ranging from T3 to L5. All pedicle screws were inserted using the fluoroscopy-assisted free-hand

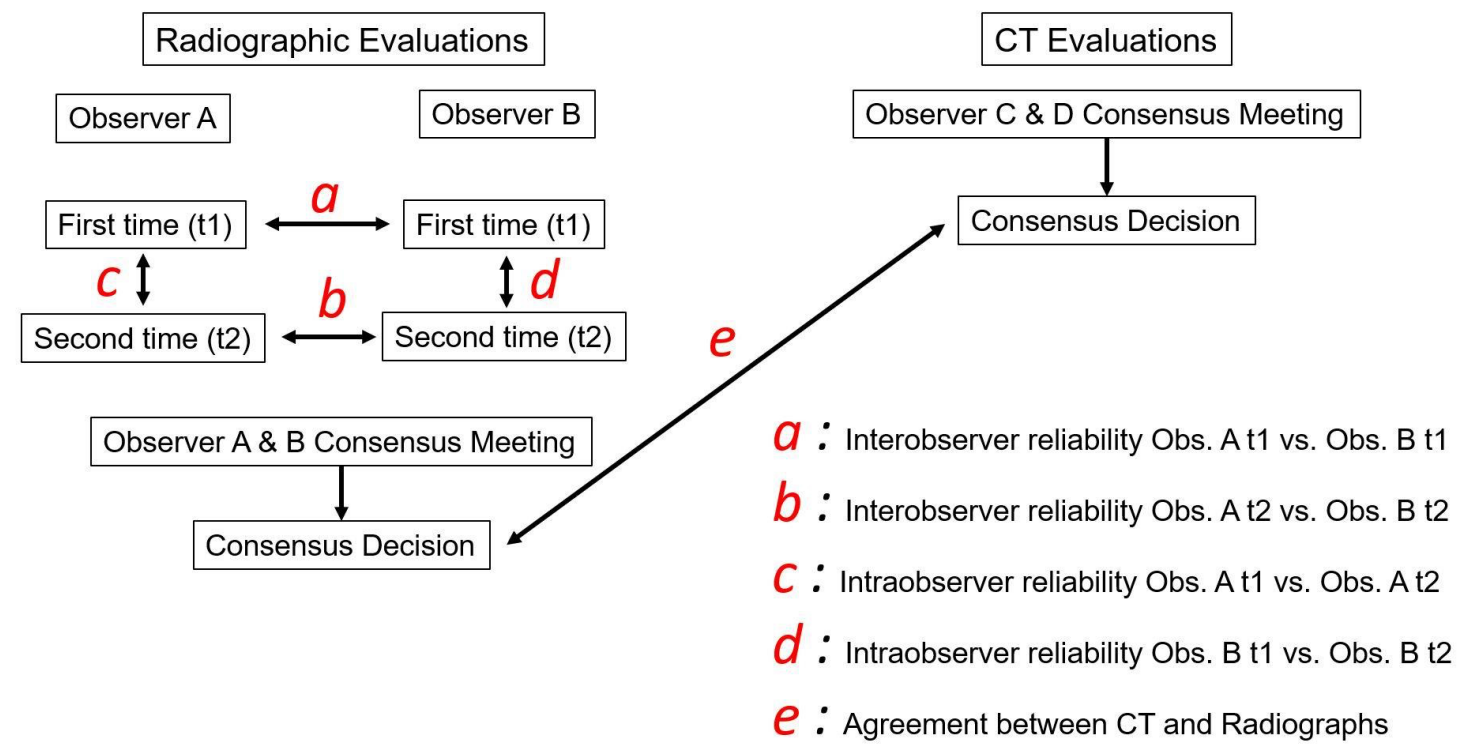

Figure 2. Diagram showing study design and statistical comparisons

$\mathrm{CT}$ : Computerized tomography, Obs.: Observer

Table 1. The number of pedicle screws and their direction in consensus decision with plain radiographs and CT

\begin{tabular}{|c|c|c|c|}
\hline & $\begin{array}{l}\text { Consensus } \\
\text { CT ratings }\end{array}$ & $\begin{array}{l}\text { Consensus } \\
\text { radiographic ratings }\end{array}$ & Accuracy \\
\hline Correct (n, \%) & 298 (81.2\%) & 314 (85.6\%) & 290 True rating (97.3\%) \\
\hline Lateral (n, \%) & $16(4.4 \%)$ & $12(3.3 \%)$ & 11 True rating (68.7\%) \\
\hline Superior (n, \%) & $1(0.3 \%)$ & $3(0.8 \%)$ & 1 True rating (100\%) \\
\hline Total (n, \%) & 367 (100\%) & 367 (100\%) & 333 True rating (90.7\%) \\
\hline
\end{tabular}


technique. None of the patients had neurovascular or visceral complications.

A total of 367 screws were evaluated. According to $\mathrm{CT}$ evaluation, 69 (18.8\%) pedicles screws were determined as malpositioned. However, in plain radiographs, 53 (14.4\%) pedicle screws were rated as malpositioned (Table 1 ). The intra-observer reliability of radiographic ratings was almost perfect for both observers at both times. On the other hand, the interobserver reliability was substantial on both occasions (Table 2). The agreement between radiographic ratings and CT evaluation (reference standard) was substantial (kappa $=0.686,95 \%$ confidence interval=0.586-0.785). The sensitivity and specificity of radiographs to detect a malpositioned screw were $65.2 \%$ and $97.3 \%$, respectively (Table 3 ).

\section{DISCUSSION}

The current study tested the intra and interobserver reliability of radiographic evaluation to detect pedicle screw malposition and its accuracy using $\mathrm{CT}$ as the reference standard. Reliability analysis showed an almost perfect intra-observer agreement; however, the interobserver agreement was under acceptable limits (kappa<0.800). More importantly, there was a significant discrepancy between radiographic ratings and $\mathrm{CT}$. The sensitivity of radiographs to detect a malpositioned pedicle screw was $65.2 \%$. In other words, radiographs cannot diagnose one of every three malpositioned pedicle screws. Anterior cortical penetration and medial wall violation are the most common inaccurate ratings with radiographic evaluation.
Although radiographic ratings were reliable, they were not entirely accurate. Thus, in case of suspicion, CT should be taken for confirmation. It should be kept in mind that both clinical and imaging findings should be evaluated together to decide on revision surgery. Many authors have reported that a slight breach on the pedicle wall is entirely asymptomatic. However, screw malposition can cause serious complications such as new radicular pain, weakness, sensory loss, more seriously, complete paralysis.

Considerably variable results regarding the reliability of conventional radiography and CT have been reported in current literature.

A possible explanation for achieving such variable results is that there is no consensus on the gold standard evaluation and/or classification method for the screw position. In a recent systematic review, Aoude et al.(15) examined 68 articles that evaluated the reliability of either conventional radiography (4 papers) or CT (64 papers). They concluded that there was no standardized method to determine the accuracy of pedicle screw position, and many of these authors did not describe their evaluation criteria ${ }^{(15)}$. Unlike these studies, the current study was conducted using predefined criteria for ratings, which was the most important strength of our study ${ }^{(12,13)}$. In addition, the direction of the malpositioned screw was included in the assessment, apart from general considerations such as "in" or "out".

The most critical weakness of the current study was the use of $\mathrm{CT}$ as a reference standard. It has been reported that the accuracy of $\mathrm{CT}$ is also controversial in cadaver studies since the

Table 2. Intra and interobserver reliability of radiographic evaluations

\begin{tabular}{|c|c|c|c|}
\hline & Карра & $95 \% \mathrm{Cl}$ & Interpretation \\
\hline \multicolumn{4}{|c|}{ Intra-observer reliability } \\
\hline Obs. $A t_{1}$ vs. Obs. $A t_{2}$ & 0.833 & $(0.814-0.951)$ & Almost perfect \\
\hline \multicolumn{4}{|l|}{ Interobserver reliability } \\
\hline Obs. A t ${ }_{1}$ vs. Obs. B t & 0.722 & $(0.622-0.821)$ & Substantial \\
\hline
\end{tabular}

Table 3. Agreement between consensus radiographic ratings and consensus CT findings

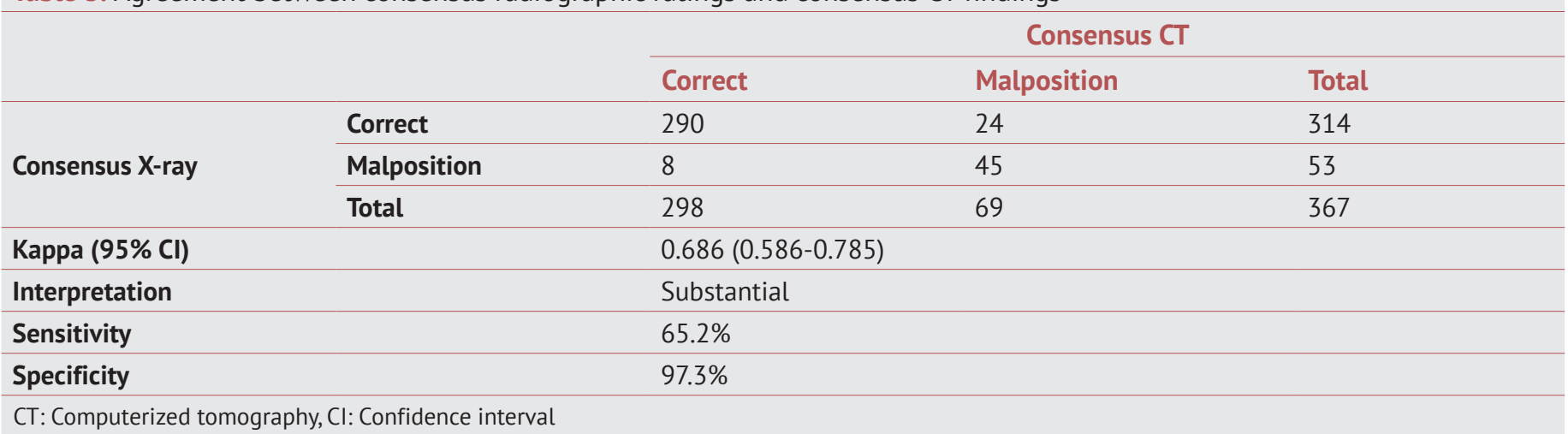


exact placement of the screw is determined by direct visual and tactile dissection ${ }^{(16-22)}$. Therefore, cadaver studies provide more reliable information regarding the accuracy of the imaging studies (Table 4). Learch et al.(16) reported that the sensitivity of conventional radiographs to detect a malpositioned screw was $63 \%$, and the CT was $87 \%$. Ferrick et al. ${ }^{(20)}$ reported that the accuracy varied between $73 \%$ to $83 \%$ depending on surgeon experience based on the classification of screw position to be inside or outside. Choma et al. ${ }^{(21)}$ found that the sensitivity of the radiographic technique was $70.1 \%$ and specificity was $83.0 \%$, whereas sensitivity for CT scans was $84.7 \%$ and specificity was $89.7 \%$. Brooks et al. ${ }^{(22)}$ showed that the sensitivity of radiographs, $\mathrm{CT}$, and combined use of modalities were $93.9 \%, 94 \%$, and $98.7 \%$, respectively, while the specificity was $12.5 \%, 36.7 \%$, and $40.7 \%$, respectively. They suggested that routine evaluation of pedicle screw postoperatively can be reliably obtained with plain radiographs, while patients who present with significant complaints of pain and/or neurologic deficits, the best way to detect correctly placed screws is with the combination of CT and plain radiography ${ }^{(22)}$. In our study, with reference to $\mathrm{CT}$, the agreement between the observers was acceptable in radiological evaluation (kappa $=0.722,0.808$ ). In addition, the sensitivity and specificity of the radiography were $65.2 \%$ and $97.3 \%$, respectively.

The use of intraoperative fluoroscopy and postoperative plain radiographs is currently the standard for screw assessment in many centers. Because, considering severe complications related to the screw malposition, intraoperative accurately determining the anatomical location of the screw provides important information to the surgeon in deciding whether the screw placement needs to be revised. However, many studies did not report their criteria for evaluating intraoperative or postoperative radiography so far. Kim et al.(12) described retrospectively based on postoperative $\mathrm{CT}$ to establish reliable and accurate criteria for intraoperative evaluating screw position. They suggest that Intraoperative plain radiographs alone using three radiographic criteria were very sensitive and accurate to detect lateral wall pedicle screw violations and also extremely specific and accurate for assessing for medial wall violations in scoliotic and kyphotic spinal deformities ${ }^{(12)}$. Weinstein et al. ${ }^{(23)}$, in which the screw position in the thoracic spine was evaluated by plain radiography, reported that $21 \%$ had medial cortical perforation, and of those $92 \%$ were in the spinal canal. They showed that the interobserver agreement was $74 \%$, the sensitivity was $31 \%$ for the radiographic evaluation of perforation, and the specificity was $90 \%$. In addition, they reported that the false-negative evaluation was $14.5 \%$, and the incorrect grading was approximately $60 \%{ }^{(23)}$.

The role of the lateral radiographs in the evaluation of screw position is controversial ${ }^{(24)}$. A true lateral radiograph alone was inaccurate to determine the penetration of the anterior cortex by the pedicle screw. Whitecloud et al.(25) examined the accuracy of the lateral radiographs in a cadaver study. To detect an anterior cortical penetration on a lateral radiograph, the $\mathrm{X}$-ray tube should be angled $5^{\circ}$ in the coronal plane for T12 - L3 levels and $10^{\circ}$ for L4 - S1 levels. They recommended additional oblique views to determine accurate screw penetration ${ }^{(25)}$ The findings in this study are consistent with our results. The anterior cortical penetration could be detected in two screws out of nine $(22.2 \%)$, since only true lateral radiographs were used in the current study.

Table 4. Previously published cadaver studies reporting the reliability of pedicle screw position ratings using direct radiography and $\mathrm{CT}$

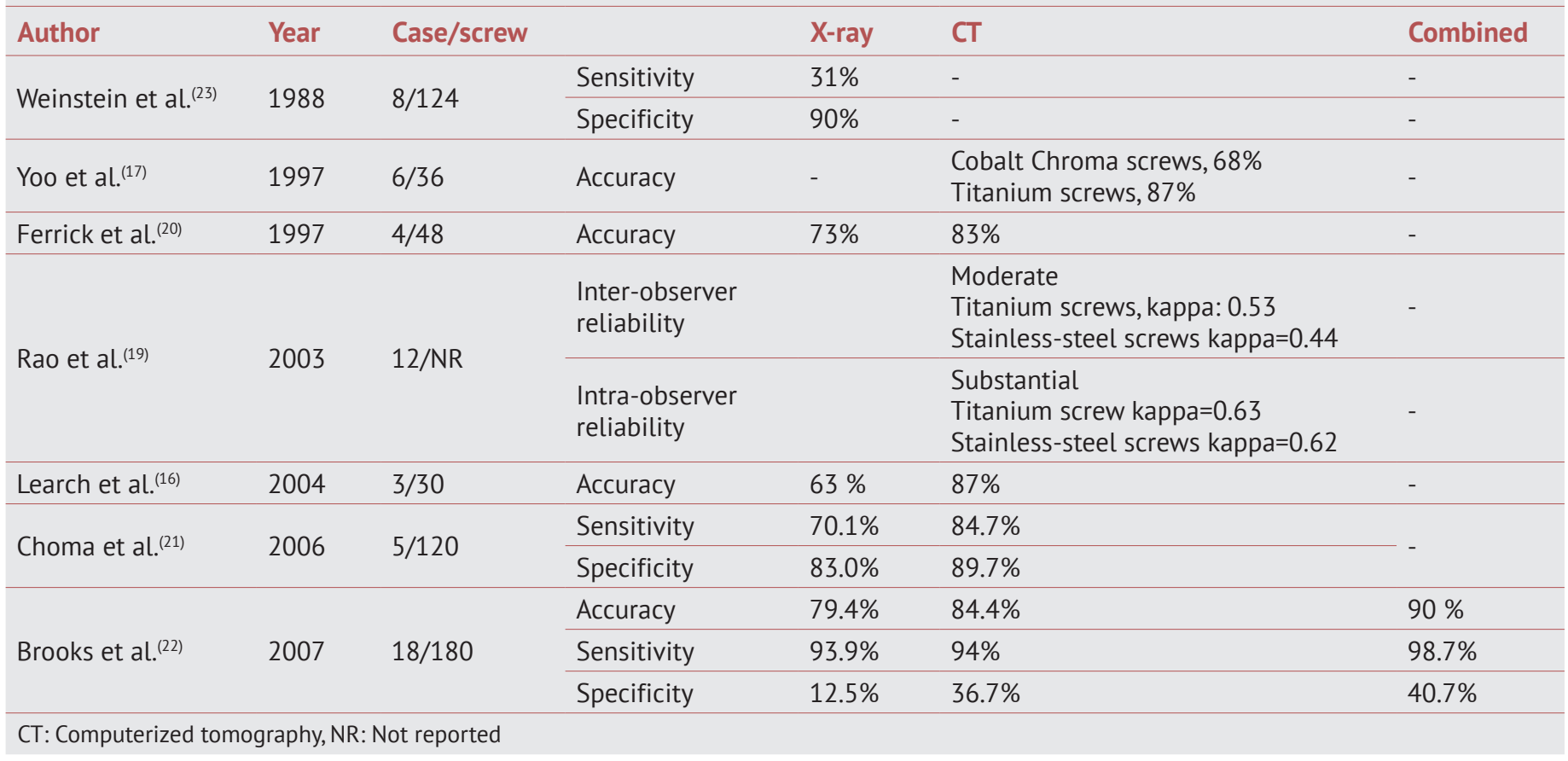




\section{Study Limitations}

There are some strengths and limitations of this study. The most important weakness of the current study was the use of CT as a reference standard, which may not reflect the correct position. Although two observers collectively rated CT scans using advanced imaging techniques, some of the screws might be incorrectly evaluated on the CT. The second limitation is the inclusion of a heterogeneous group of patients with different etiologies. Determination of the midline of the vertebrae might be faulty in patients with rotational deformity.

\section{CONCLUSION}

In the evaluation of postoperative pedicle screw placement, plain AP and lateral radiographs may be insufficient, particularly determination of medial and anterior violation. Although the agreement of observers was within the acceptable limits, the accuracy was low compared to CT evaluation. CT should be used to evaluate the accuracy of screw position in cases such as patients with postoperative neurological deficits.

\section{REFERENCES}

1. Braga BP, de Morais JV, Vilela MD. Free-hand placement of high thoracic pedicle screws with the aid of fluoroscopy: evaluation of positioning by CT scans in a four-year consecutive series. Arq Neuropsiquiatr. 2010;68:390-5.

2. Gautschi OP, Schatlo B, Schaller K, Tessitore E. Clinically relevant complications related to pedicle screw placement in thoracolumbar surgery and their management: a literature review of 35,630 pedicle screws. Neurosurg Focus. 2011;31:E8. doi: 10.3171/2011.7.FOCUS11168.

3. Chan CYW, Kwan MK. Safety of pedicle screws in adolescent idiopathic scoliosis surgery. Asian Spine J. 2017;11:998-1007.

4. Perdomo-Pantoja A, Ishida W, Zygourakis C, Holmes C, lyer RR, Cottrill $E$, et al. Accuracy of Current Techniques for Placement of Pedicle Screws in the Spine: A Comprehensive Systematic Review and MetaAnalysis of 51,161 Screws. World Neurosurg. 2019;126:664-78.

5. Kayacı S, Cakir T, Dolgun M, Cakir E, Bozok Ş, Temiz C, Caglar YS. Aortic injury by thoracic pedicle screw. When is aortic repair required? literature review and three new cases. World Neurosurg. 2019;128:216-24.

6. Esses SI, Sachs BL, Dreyzin V. Complications associated with the technique of pedicle screw fixation. Spine (Phila Pa 1976). 1993; 18:2231-9.

7. West JL 3rd, Ogilvie JW, Bradford DS. Complications of the variable screw plate pedicle screw fixation. Spine. 1991;16:576-9.

8. Sarlak AY, Tosun B, Atmaca H, Sarisoy HT, Buluç L. Evaluation of thoracic pedicle screw placement in adolescent idiopathic scoliosis. Eur Spine J. 2009;18:1892-7.
9. Açikbaş SC, Arslan FY, Tuncer MR. The effect of transpedicular screw misplacement on late spinal stability. Acta Neurochir (Wien). 2003;145:949-54; discussion 954-5.

10. Zheng X, Qian BP, Liu Z, Sun X, Zhu ZZ, Wang B, et al. Screw Placement at the Apex Alters Surgical Outcomes of Moderate Lenke 1 Adolescent Idiopathic Scoliosis. Clin Spine Surg. 2017;30:E883-91. doi: $10.1097 / B S D .0000000000000435$.

11. Temel G, Erdogan S. Sample size in agreement studies. Marmara Med J. 2017;30:101-12.

12. Kim YJ, Lenke LG, Cheh G, Riew KD. Evaluation of pedicle screw placement in the deformed spine using intraoperative plain radiographs: a comparison with computerized tomography. Spine (Phila Pa 1976). 2005;30:2084-8.

13. Polat B, Polat AE, Atıcı Y, Karaguven D, Erdogan S, Benli IT. Evaluation of screw malposition by radiography in Lenke type 1 adolescent idiopathic scoliosis patients having undergone selective fusion. J Turk Spinal Surg. 2016; 27:219-24.

14. Landis JR, Koch GG. The measurement of observer agreement for categorical data. Biometrics.1977;33:159-74.

15. Aoude AA, Fortin M, Figueiredo R, Jarzem P, Ouellet J, Weber MH Methods to determine pedicle screw placement accuracy in spine surgery: a systematic review. Eur Spine J. 2015;24:990-1004.

16. Learch TJ, Massie JB, Pathria MN, Ahlgren BA, Garfin SR. Assessment of pedicle screw placement utilizing conventional radiography and computed tomography: a proposed systematic approach to improve accuracy of interpretation. Spine (Phila Pa 1976). 2004; 29:767-73.

17. Yoo JU, Ghanayem A, Petersilge C. Accuracy of using computed tomography to identify pedicle screw placement in cadaveric human lumbar spine. Spine (Phila Pa 1976). 1997;22:2668-7.

18. Castro WHM, Halm H, Jerosch J,Malms J, Steinbeck J, Blasius S. Accuracy of pedicle screw placement in lumbar vertebrae. Spine (Phila Pa 1976).1996;21:1320-4.

19. Rao G, Brodke DS, Rondina M, Bacchus K, Dailey AT. Inter- and intraobserver reliability of computed tomography in assessment of thoracic pedicle screw placement. Spine (Phila Pa 1976). 2003;28:2527-30.

20. Ferrick MR, Kowalski JM, Simmons ED. Reliability of roentgenogram evaluation of pedicle screw position. Spine. 1997;22:1249-53.

21. Choma TJ, Denis F, Lonstein JE, Perra JH, Schwender JD, Garvey TA, et al. Stepwise methodology for plain radiographic assessment of pedicle screw placement: a comparison with computed tomography. J Spinal Disord Tech. 2006;19:547-53.

22. Brooks D, Eskander M, Balsis S, Ordway N, Connolly P. Imaging assessment of lumbar pedicle screw placement: sensitivity and specificity of plain radiographs and computer axial tomography. Spine (Phila Pa 1976). 2007;32:1450-3.

23. Weinstein JN, Spratt KF, Spengler D, Brick C, Reid S. Spinal pedicle fixation: Reliability and validity of roentgenogram-based assessment and surgical factors on successful screw placement. Spine (Phila Pa 1976). 1988;13:1012-8.

24. Odgers CJ 4th, Vaccaro AR, Pollack ME, Cotler JM. Accuracy of pedicle screw placement with the assistance of lateral plain radiography. J Spinal Disord. 1996;9:334-8.

25. Whitecloud TS, Skalley TC, Cook SD, Morgan EL. Roentgenographic measurement of pedicle screw penetration. Clin Orthop Relat Res. 1989;245:57-68. 